\title{
Glass-Coated Beryllium Mirrors for the LHCb RICH1 Detector
}

\author{
G.J. Barber ${ }^{a}$, N.H. Brook ${ }^{b}$, W. Cameron ${ }^{a}$, C. D'Ambrosio ${ }^{c}$, C. Frei ${ }^{c}$, \\ N. Harnew ${ }^{d}$, R. Head ${ }^{b}$, Y.P. Khimitch ${ }^{e}$, V. Khmelnikov ${ }^{f}$, \\ P. Loveridge ${ }^{g}$, F.C.D. Metlica ${ }^{b}$, V. Obraztsov ${ }^{f}$, D. Piedigrossi ${ }^{c}$, \\ V. Sizenev ${ }^{h}$, P.M. Szczypka ${ }^{b}$, O. Ullaland ${ }^{c}$, E. Vygosky $^{h}$, D. Websdale ${ }^{a}$
}

April 12, 2006

\author{
${ }^{a}$ Imperial College, London, UK \\ ${ }^{b}$ University of Bristol, Bristol, UK \\ ${ }^{c}$ CERN, Geneva, Switzerland \\ ${ }^{d}$ University of Oxford, Oxford, UK \\ ${ }^{e}$ Vavilov State Optical Institute, St. Petersburg, Russia \\ ${ }^{f}$ Institute for High Energy Physics (IHEP-Serphukov), Protvino, Russia \\ ${ }^{g}$ Rutherford Appleton Laboratory, Chilton, UK \\ ${ }^{h}$ Kompozit Joint Stock Company, Moscow, Russia
}

\begin{abstract}
The design, manufacture and testing of lightweight glass-coated beryllium spherical converging mirrors for the RICH1 detector of $\mathrm{LHCb}$ are described. The mirrors need to be lightweight to minimize the material budget and fluorocarbon-compatible to avoid degradation in the RICH1 $\mathrm{C}_{4} \mathrm{~F}_{10}$ gas radiator. Results of the optical measurements for the small-sized prototypes and for the first full-sized prototype mirror are reported.
\end{abstract}




\section{Introduction}

The LHCb experiment [1], [2] is designed to study B decays and CP violation at the CERN Large Hadron Collider, LHC. The experiment has a Ring Imaging Cherenkov (RICH) detector system [3], that will provide a powerful particle identification tool. The RICH system uses two detectors, RICH1 and RICH2 to cover the momentum range 1-100 GeV/c. In RICH1, the focusing of Cherenkov light onto the photon detectors is achieved using a combination of spherical converging mirrors which lie within the detector acceptance and secondary planar mirrors positioned outside the detector acceptance. The spherical mirrors must be lightweight to minimize the amount of material within the detector acceptance and must be stable in the RICH1 $\mathrm{C}_{4} \mathrm{~F}_{10}$ fluorocarbon gas radiator environment.

Over recent years, the need to manufacture lightweight large sized mirror optics for space telescopes led to research with other optical materials to replace glass. Beryllium $(Z=4)$ looked very promising as an alternative material because of its unique properties, ideal for lightweight applications requiring high rigidity. Several beryllium mirrors [4], [5], [6] have been built successfully for space applications.

The idea of using beryllium type mirrors for RICH1 was proposed by Tom Ypsilantis. Over the past years starting from 1998 [7], two technologies were tested in parallel for the spherical mirrors of RICH1: composite ${ }^{1}$ and glass-coated beryllium. The composite mirrors proved to be unstable and degraded over time in a fluorocarbon environment, in contrast to the beryllium mirrors which were tested successfully. Hence beryllium technology was chosen for the RICH1 mirrors.

Beryllium's main properties [9], [10] are a long radiation length $\left(X_{\circ}\right)$, lightweight, nonmagnetic, good rigidity (Young's Modulus, $E$ ), fluorocarbon compatibility, high melting point $\left(1285^{\circ} \mathrm{C}\right)$, low coefficient of thermal expansion $(\alpha)$, and oxidation resistance in air. A comparison between the basic properties of materials which can be used to manufacture lightweight mirrors is given in Table 1. The principal disadvantages of beryllium are its high toxicity which requires special safety measures during manufacturing and particular care when handling the mirror, and the higher manufacturing cost when compared to other mirror technologies.

Polished beryllium surfaces are typically only $\sim 50 \%$ reflective in the visible and ultraviolet range. A thin glass layer fused onto a beryllium substrate provides a glass surface which can be polished by standard optical methods and then coated with an aluminum reflective film, increasing the mirror reflectivity to $\sim 90 \%$. The beryllium substrate serves to support

\footnotetext{
${ }^{1}$ Two types of composite mirrors were tested. The first type (polymethyl-metacrylate based) was manufactured in house [8], while the second (carbon-fiber epoxy) was manufactured commercially.
} 
the thin glass layer, being rigid enough to maintain the desired reflective spherical surface. The thermal expansion of the glass can be tuned to match that of the beryllium.

\begin{tabular}{|c|c|c|c|c|c|}
\hline Material & $\begin{array}{c}X_{\circ} \\
\left(\mathrm{g} / \mathrm{cm}^{2}\right)\end{array}$ & $\begin{array}{c}\lambda_{I} \\
\left(\mathrm{~g} / \mathrm{cm}^{2}\right)\end{array}$ & $\begin{array}{c}E \\
(\mathrm{GPa})\end{array}$ & $\begin{array}{c}\alpha \\
\left(10^{-6} /{ }^{\circ} \mathrm{C}\right)\end{array}$ & $\begin{array}{c}\rho \\
\left(\mathrm{g} / \mathrm{cm}^{3}\right)\end{array}$ \\
\hline \hline Pyrex (borosilicate glass) & 28.1 & $\approx 97$ & 64 & 3.2 & 2.23 \\
\hline \hline Beryllium & 65.19 & 75.2 & 255 & 12.4 & 1.848 \\
\hline \hline Aluminum & 24.01 & 106.4 & 69 & 23.9 & 2.70 \\
\hline \hline Plexiglas & 40.49 & 83.0 & 3.3 & 70 & 1.19 \\
\hline
\end{tabular}

Table 1: Properties of typical materials [11] used to construct lightweight mirrors: radiation length $X_{\circ}$, interaction length $\lambda_{I}$, Young's Modulus $E$, coefficient of thermal expansion $\alpha$ and density $\rho$. Pyrex consists principally of $\mathrm{SiO}_{2}(\sim 80 \%)$ which has $\lambda_{I}=97.4 \mathrm{~g} / \mathrm{cm}^{2}$.

\section{Mirror Characterization}

The two main parameters defining the optical quality of a mirror are the mirror radius of curvature $R$ and the average geometrical quality $D_{\circ} . D_{\circ}$ is defined as the diameter of the circle at the mirror centre of curvature ( $\mathrm{CoC}$ ) which contains $95 \%$ of the reflected light intensity from a point source placed at the CoC. The setup for the measurement of spherical converging mirrors [12], [13] is described in the Appendix.

The precision of the $D_{\circ}$ and $R$ measurements are $\sigma_{D_{\circ}}=0.06 \mathrm{~mm}$ and $\sigma_{R}=1 \mathrm{~mm}$, respectively. The quantity $\sigma_{s}$, defined as $\sigma_{s}:=D_{\circ} / 4$, would represent the rms value of the spot light distribution if it had a Gaussian shape. The angular precision of the mirror, $\sigma_{\theta}$, is defined as the rms angular deviation of the normal to the mirror surface at a given point from the radius of curvature at that point and is related to $D_{\circ}$ :

$$
\sigma_{\theta}:=\frac{\sigma_{s}}{2 R}=\frac{D_{\circ}}{8 R}
$$

where the factor 2 in the denominator takes account of the reflection at the mirror surface. $D_{\circ}$ is independent of the spot shape and distribution; while $\sigma_{\theta}$, where a radial symmetry for the spot is supposed, can be considered only as an approximation of the rms of the spot distribution. 


\section{$3 \quad$ Small-Sized Prototype Beryllium Mirrors}

Three small-sized glass-coated beryllium mirror prototypes were manufactured in Russia ${ }^{2}$ and were tested at CERN [12], during the years 1998 to 2001. Photographs of the prototypes are shown in Fig. 1. The first prototype is simply a circular flat mirror, while the other two prototypes are spherical converging mirrors.
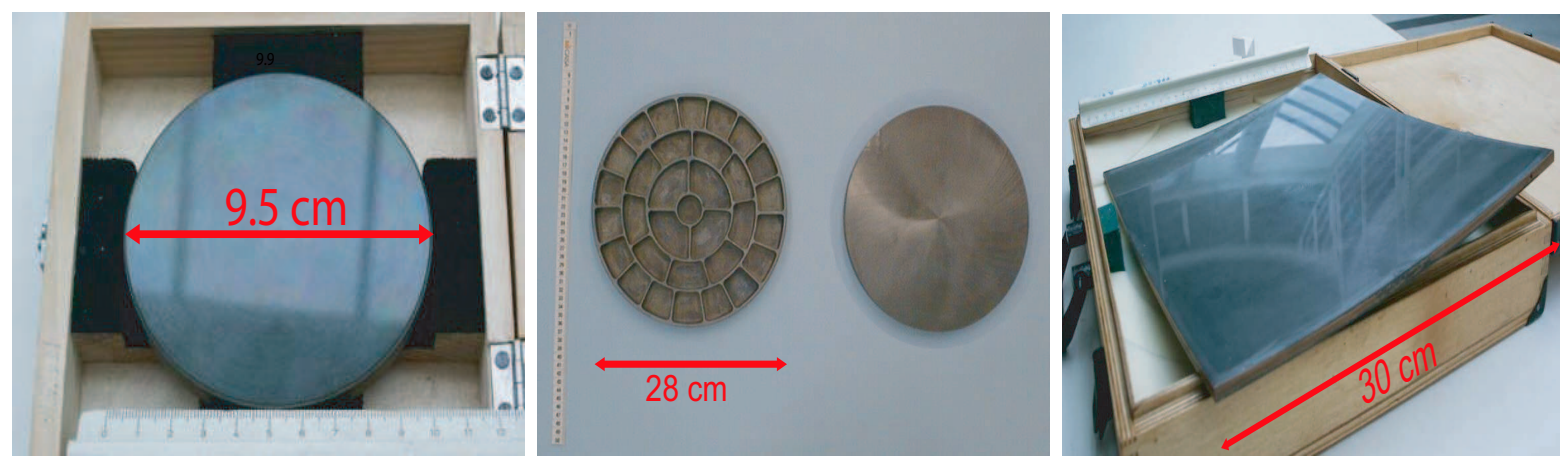

Figure 1: Photographs of the small-sized prototype glass-coated beryllium mirrors. The left photo is a front view of prototype 1 (flat mirror); the central image shows the back side (left) with the reinforcing rib structure and the front side (right) of prototype 2; the right photo is a front view of prototype 3.

The flat mirror (prototype 1) has a diameter of $95 \mathrm{~mm}$ and consists of a $10 \mathrm{~mm}$ thick beryllium substrate coated with a $1 \mathrm{~mm}$ glass layer, resulting in $3.6 \% X_{\circ}$ and a $D_{\circ}$ measured $^{3}$ to be less than $0.1 \mathrm{~mm}$.

The second prototype is also circular, with a diameter of $280 \mathrm{~mm}$, consisting of a $5 \mathrm{~mm}$ thick beryllium substrate with a $1 \mathrm{~mm}$ glass layer coating and reinforced by a $20 \mathrm{~mm}$ thick rib-like structure. The average thickness is $3.3 \% X_{\circ}$, with $7.9 \% X_{\circ}$ at the ribs and $2.2 \% X_{\circ}$ between the ribs. The measured mirror precision is very high, $\sigma_{\theta}=0.013 \mathrm{mrad}$ ( $D_{\circ}=0.85 \mathrm{~mm}$ ), and the measured radius of curvature $R=7926 \mathrm{~mm}$ is close to the design value of $R=8000 \mathrm{~mm}$. The rib-like structure ensures high mirror rigidity.

After the successful testing of the first two prototypes, a third prototype was manufactured according the LHCb RICH1 specifications of the time [1], $\mathrm{R}=1700 \mathrm{~mm}$ and $D_{\circ}<2 \mathrm{~mm}$. The third prototype is rectangular, $375 \mathrm{~mm} \times 300 \mathrm{~mm}$, consisting of a $6 \mathrm{~mm}$ thick beryllium substrate without any rib reinforcement with a $0.3 \mathrm{~mm}$ glass coating, giving

\footnotetext{
${ }^{2}$ Developed and manufactured by Kompozit in Moscow (4 Pionerskaya St, Korolev 141070).

${ }^{3}$ The setup to measure the flat mirrors $D_{\text {。 }}$ needs to be modified to include a high quality spherical reference mirror with a very small $D_{\circ}(\sim 0.2 \mathrm{~mm})$ to focus the light, small enough to allow measurement of the $D_{\circ}$ of the flat mirror.
} 


\begin{tabular}{|c|c|c|c|c|c|c|c|}
\hline Mirror & $\begin{array}{c}\text { Dimensions } \\
(\mathrm{mm})\end{array}$ & $\begin{array}{c}\text { Thickness } \\
(\mathrm{mm})\end{array}$ & $\begin{array}{c}\mathrm{R} \\
(\mathrm{mm})\end{array}$ & $\begin{array}{c}D_{\circ} \\
(\mathrm{mm})\end{array}$ & $\begin{array}{c}\sigma_{\theta} \\
(\mathrm{mrad})\end{array}$ & $\begin{array}{c}\text { weight } \\
(\mathrm{kg})\end{array}$ & $\begin{array}{c}X_{\circ} \\
(\%)\end{array}$ \\
\hline \hline Prototype 1 & $\varnothing 95$ & 11 & $\infty$ & $<0.1$ & - & 0.15 & 3.6 \\
\hline \hline Prototype 2 & $\varnothing 280$ & 6 to 26 & 7926 & 0.85 & 0.013 & 0.95 & 3.3 \\
\hline \hline Prototype 3 & $375 \times 300$ & 6.3 & 1696 & 0.41 & 0.030 & 1.3 & 1.9 \\
\hline
\end{tabular}

Table 2: Measured parameters for the three small-sized prototype glass-coated beryllium mirrors.

$1.9 \% X_{\circ}$. It also has a good precision, $\sigma_{\theta}=0.03 \mathrm{mrad}\left(D_{\circ}=0.41 \mathrm{~mm}\right)$ and $R=1696 \mathrm{~mm}$ is very close to the design value of $1700 \mathrm{~mm}$.

Table 2 summarizes the measured parameters for the three mirrors and Fig. 2 shows the measured $D_{\circ}$. As a result of the success of these small-sized prototypes which proved the technology, a full-sized prototype mirror was designed and manufactured.


Figure 2: The measured $D_{\circ}$ for the second (top plot) and third (bottom plot) prototypes. The plots show the relative amount of the reflected light intensity (\%) contained inside circles of varying diameters. 


\section{Design of a Full-Sized Prototype Mirror}

The full-sized prototype mirror was designed to be as thin as possible, minimizing the $X_{\circ} \%$, but at the same time rigid enough not to deform under its own weight. It was designed according to the RICH1 specifications, so that if successful, it could be installed as a final RICH1 mirror. The mirror dimensions were constrained by limitations in the manufacturing size of the beryllium blanks ${ }^{4}$. The largest beryllium blank from which a mirror could be manufactured was disc shaped, $800 \mathrm{~mm}$ in diameter.

The design consists of a $3 \mathrm{~mm}$ thick spherically shaped beryllium substrate $(\mathrm{R}=2700 \mathrm{~mm})$ coated with a thin glass layer $(0.3-0.5 \mathrm{~mm})$, having a $20 \mathrm{~mm}$ thick beryllium rim at one edge to support the mirror. The shape of the mirror is approximately rectangular. The rim serves to bolt the mirror to the mirror support structure which lies outside the $\mathrm{LHCb}$ acceptance. The mirror specifications are listed in Table 3.

\begin{tabular}{|c|c|c|c|c|c|c|}
\hline $\begin{array}{c}\text { Dimensions } \\
(\mathrm{mm})\end{array}$ & $\begin{array}{c}\text { Average Thickness } \\
(\mathrm{mm})\end{array}$ & $\begin{array}{c}\mathrm{R} \\
(\mathrm{mm})\end{array}$ & $\begin{array}{c}D_{\circ} \\
(\mathrm{mm})\end{array}$ & $\begin{array}{c}X_{\circ} \\
(\%)\end{array}$ & $\begin{array}{c}\lambda_{I} \\
(\%)\end{array}$ & $\begin{array}{c}\text { Roughness } \\
(\mathrm{nm})\end{array}$ \\
\hline \hline $383.5 \times 660$ & $3(\mathrm{Be})+0.3-0.5$ (glass) & $2700 \pm 1 \%$ & $<2.5$ & $<2$ & $<1$ & $<5$ \\
\hline
\end{tabular}

Table 3: RICH1 design specifications for the full-sized prototype glass-coated beryllium mirror.

The thickness of the beryllium substrate $(3 \mathrm{~mm})$ and of the glass coating $(0.3-0.5 \mathrm{~mm})$ is to satisfy the material budget within the LHCb acceptance, i.e. $X_{\circ}<2 \%$ and $\lambda_{I}<1 \%$. The radius of curvature precision $\sigma_{R}$ should be better than:

$$
\sigma_{R} \simeq \frac{\sigma_{d}}{r_{c}} R \simeq 1.6 \% \cdot R
$$

were $\sigma_{d}(\simeq 0.72 \mathrm{~mm})$ is the photodetector precision $(2.5 \mathrm{~mm} \times 2.5 \mathrm{~mm}$ pixel size $)$ and $r_{c}$ is the maximum Cherenkov cone base radius on the mirror ( $\simeq 45 \mathrm{~mm}$ for $\mathrm{C}_{4} \mathrm{~F}_{10}$ in RICH1). A radius of curvature of $\mathrm{R} \pm 1 \%$ ensures proper focusing of the Cherenkov photons onto the

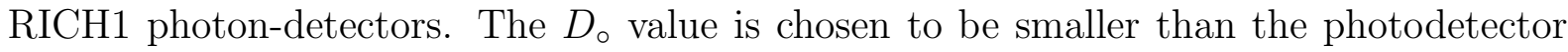
pixel size $(<2.5 \mathrm{~mm})$ to ensure a good mirror angular resolution $\left(\sigma_{\theta}<0.12 \mathrm{mrad}\right)$ which

\footnotetext{
${ }^{4}$ Due to this limitation, RICH1 was designed to have eight spherical mirrors instead of two large ones, arranged in two spherical surfaces: one above and one below the beamline. Each surface consisted of an array of four beryllium-glass mirrors and each mirror was to be supported from outside the $\mathrm{LHCb}$ acceptance. The four inner mirrors which surround the beamline were designed to be slightly larger $(407 \mathrm{~mm} \times 660 \mathrm{~mm})$ than the four outer mirrors $(383.5 \mathrm{~mm} \times 660 \mathrm{~mm})$. The prototype is an outer mirror.
} 
will contribute negligibly to the total RICH1 single photon Cherenkov angle resolution $\left(\sigma_{C h}^{t o t} \simeq 1.6 \mathrm{mrad}\right)$. In order to minimize scattering of the reflected light, the smoothness of the polished glass surface is required to be $\sim \lambda / 100$, which corresponds to a surface roughness $<5 \mathrm{~nm}$ for the wavelength region of interest $\lambda \sim 200 \mathrm{~nm}$ to $600 \mathrm{~nm}$.

A finite element analysis (FEA) [14] was performed using $\mathrm{ANSYS}^{5}$ to investigate the support scheme for the mirror in the RICH1 setup, the mirror distortion due to gravity for different support schemes, the stresses and displacements induced by thermal expansion, and bolt preload and misalignment of the mating surfaces (see Fig. 3).
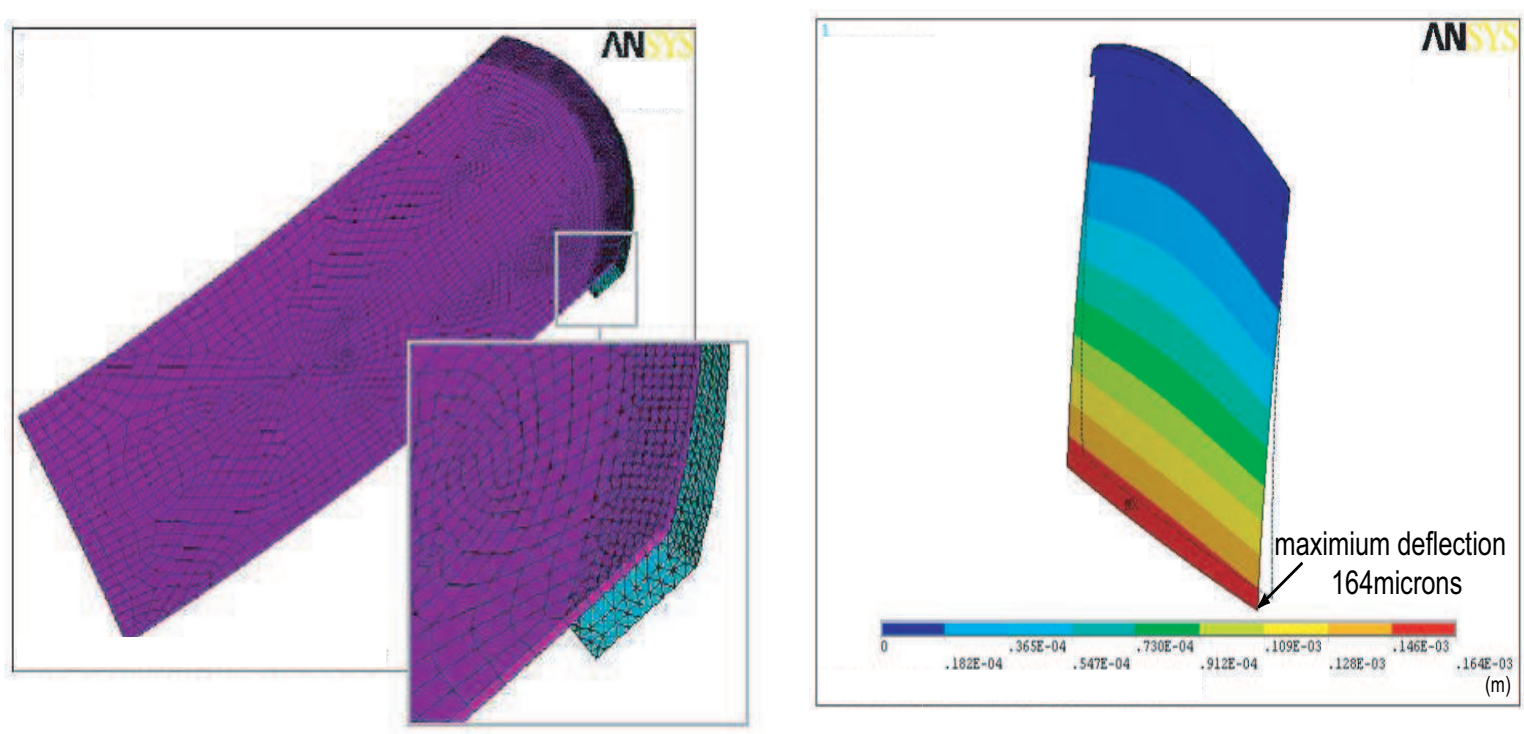

Figure 3: The left picture shows the mesh model of the mirror beryllium substrate used for the FEA. The right picture is the deflection contour plot for the mirror supported from the top by a single pin (10 $\mathrm{mm}$ diameter and no protrusion) with a vertical tilt of $12^{\circ}$ (mirror CoC pointing upwards). The maximum deflection of $164 \mu \mathrm{m}$ occurs at the bottom of the mirror.

For the FEA studies, only the beryllium substrate is considered. The results show that the compression of the rim due to the bolt preload is unlikely to cause any significant mirror deformation. For a double point support system, a careful design of the support scheme would be necessary to minimize distortions due to the differential thermal expansion between the mirror rim and the support structure, and the possible misalignment of the mating surfaces.

\footnotetext{
${ }^{5}$ General-purpose finite element analysis (FEA) computer aided engineering software tools developed by ANSYS, Inc.
} 
A central single point support system was chosen because it is the simplest support scheme and avoids complications of a multiple point support system. Various combinations of pin diameters and lengths were studied. The mirror was held tilted by $12^{\circ}$ vertically (Fig. 3), which corresponds to one of the two possible tilts of the mirror in RICH1. A single point support consisting of a $10 \mathrm{~mm}$ diameter pin with no protrusion beyond the mirror rim gives the least deflection. The maximum deflection of the mirror surface is $164 \mu \mathrm{m}$ compared to an $83 \mu \mathrm{m}$ maximum deflection for the best case possible where the mirror is supported continously along the rim.

For the opposite case where the mirror is supported by its rim from the bottom, and held tilted vertically ${ }^{6}$ by $12^{\circ}$, the resulting magnitude of the deflection is the same but the direction is reversed. Also the five lowest vibration modes were calculated: $21.4 \mathrm{~Hz}$, $59.4 \mathrm{~Hz}, 72.7 \mathrm{~Hz}, 121.3 \mathrm{~Hz}$, and $199.4 \mathrm{~Hz}$. These lie away from the typical $50 \mathrm{~Hz}$ mechanical vibration of electric equipment which might be present in the LHCb experimental area.

The FEA surface displacements of the mirror due to gravity were used to calculate the variation in the radius of curvature for elemental areas on the mirror surface by a fitting procedure, to obtain the approximate effect on the mirror $D_{\circ}$. The fitting procedure involves looping over each FEA point on the mirror surface to find the four nearest neighbours and then fitting them to a sphere. Each FEA point is weighted by the local surface density of FEA points. The results are shown in Fig. 4. In the y-direction (vertical) the mean shift is $\sim 700 \mu \mathrm{m}$ with an RMS of $\sim 250 \mu \mathrm{m}$, while in the $\mathrm{x}$-direction (horizontal, parallel to the rim) and in the z-direction the mean shift of the centre of curvature and RMS are similar, i.e. $\sim 20 \mu \mathrm{m}$ and RMS of $\sim 120 \mu \mathrm{m}$. This corresponds to a slight increase of the mirror radius of curvature and widening of the spot at the centre of curvature. This is still within the RICH1 tolerances and proves the feasibility of the mirror design.

Three drawings of the mirror are shown in Fig. 5 and Fig. 6. The mirror rim has three holes into which titanium inserts are glued; this is in accordance with the beryllium safety rules which prohibit any direct fixing of bolts to the beryllium bulk. The central hole supports the mirror from the top (bottom) and is bolted to the support structure. Two pins fixed to the support bar are inserted into the side holes as a safety mechanism to prevent rotation of the mirror about the central hole axis in case of accidental shocks. The outer part of the rim is cut to form a flat surface, so that when the mirror is bolted to its support bar, it is held at the required angle in RICH1.

\footnotetext{
${ }^{6}$ For this case the mirror centre of curvature is pointing downwards.
} 


\section{Entries}



\section{Entries}
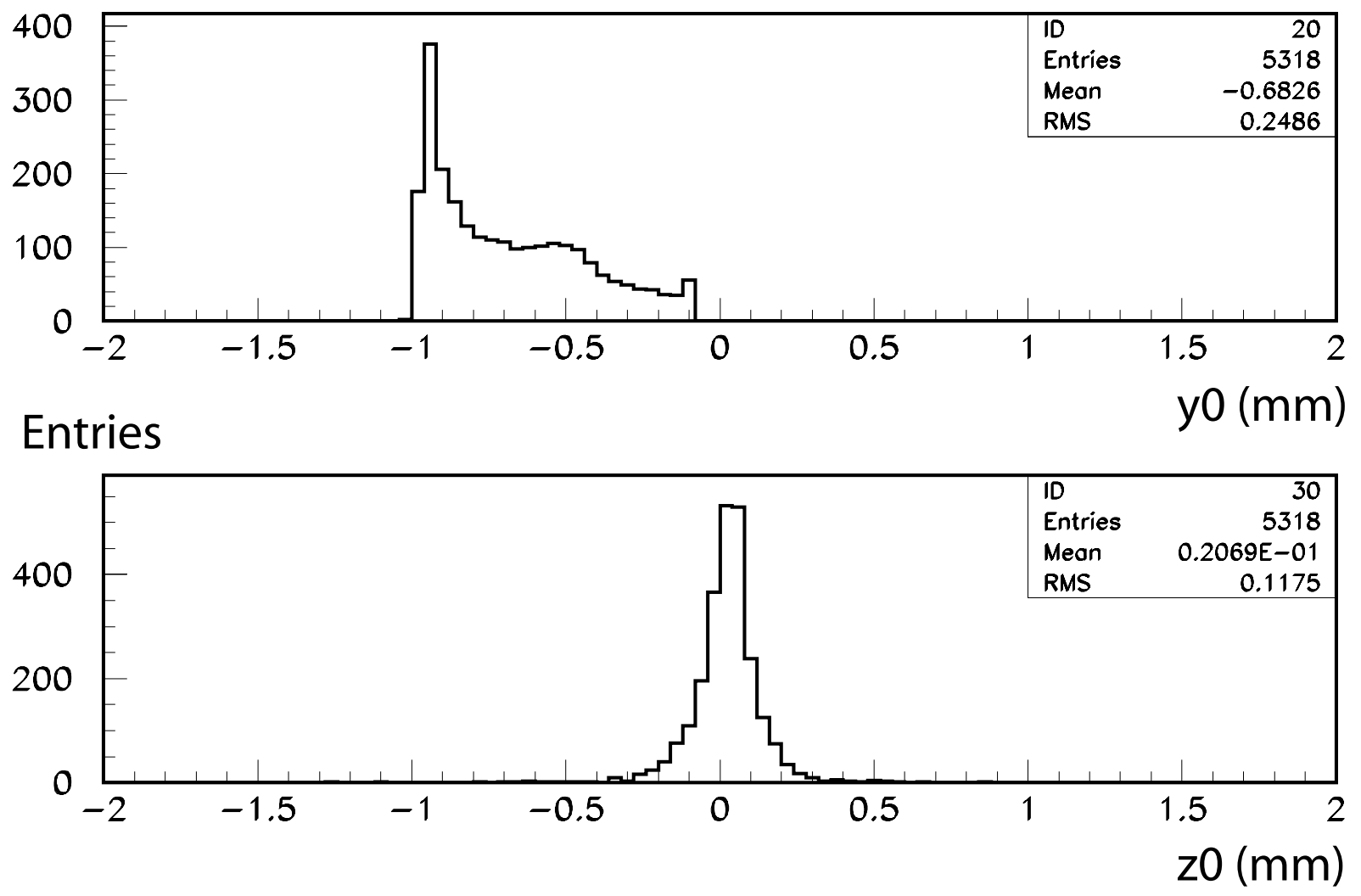

Figure 4: The distribution of coordinates $(\mathrm{mm})$ of the centre of curvature of elemental areas of the mirror surface. The coordinates $\mathrm{x} 0=0, \mathrm{y} 0=0, \mathrm{z} 0=0$ correspond to the position of the centre of curvature for the undeformed mirror (no gravity applied). In the $y$ direction the mean shift is $\sim 700 \mu \mathrm{m}$ with an RMS of $\sim 250 \mu \mathrm{m}$, while in the $\mathrm{x}$-direction and in the $\mathrm{z}$-direction the mean shift of the centre of curvature and RMS are similar, i.e. $\sim 20 \mu \mathrm{m}$ and $R M S$ of $\sim 120 \mu \mathrm{m}$. 


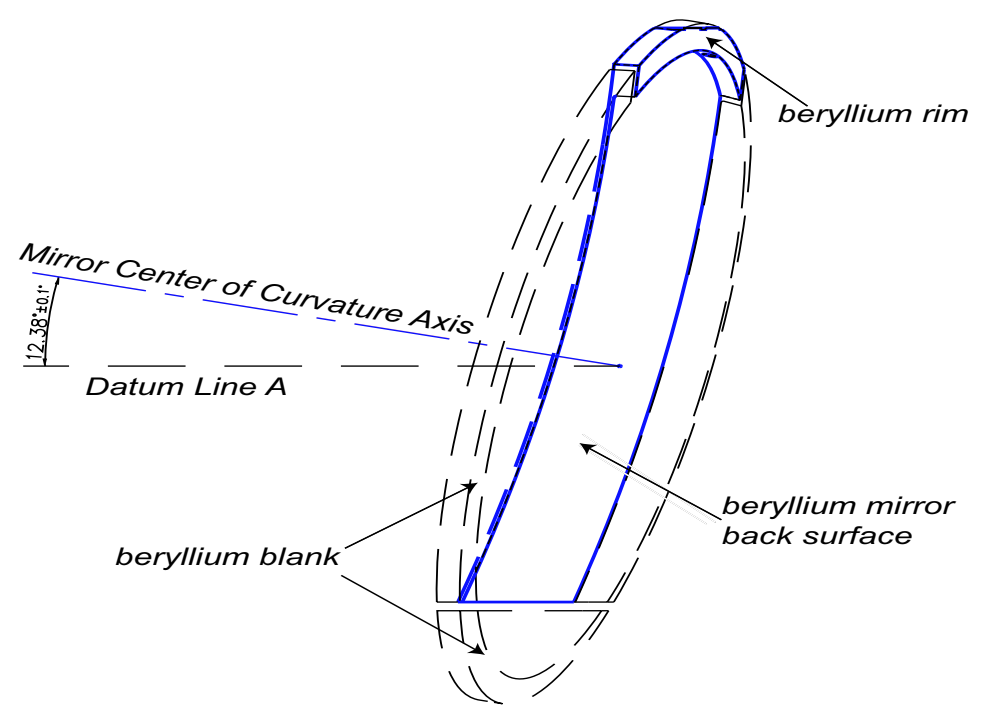

Figure 5: A drawing showing the outline of the mirror within the disk-shaped blank from which the mirror is machined. Datum Line $A$ is the horizontal axis.


Figure 6: Two drawings of the mirror, side view (left) and top view (right). The mirror overall dimensions and the angular orientation are shown. The mirror vertical tilt is $12.38^{\circ}$ i.e. the angle between the mirror centre of curvature axis (projection onto plane of this page) and the horizontal (Datum Line A). The mirror horizontal tilt is $12.97^{\circ}$ i.e. the angle between the mirror centre of curvature axis (projection onto Datum plane $B$ ) and the horizontal (Datum Line A). The rim is cut to form a flat surface for mounting the mirror with the required horizontal and vertical tilt. The web-like line structure in the drawings serves only to guide the eye. 


\section{Manufacture of a Full-Sized Prototype Mirror}

The full-sized prototype mirror was ordered from Russia in July 2004. It arrived in CERN a year later in July 2005 because of the extensive R\&D necessary in manufacturing such a thin large-sized beryllium-glass mirror. The mirror was manufactured by Kompozit ${ }^{7}$ in collaboration with the Vavilov State Optical Institute ${ }^{8}$ under the supervision and coordination of IHEP-Protvino. The beryllium was procured from Ulba ${ }^{9}$ in Kazakhstan. An overview of the manufacturing process is given in the following paragraphs, however the details are propriety information.

The so-called "vacuum-hot-pressing" technology is used to produce the beryllium blank at Ulba. Powder metallurgy is used to manufacture beryllium parts (see Appendix). Beryllium powder is placed into a disc-shaped die where the powder is vibrated to obtain a homogeneous distribution, while heat and pressure are applied to compress and consolidate the powder into a solid metallic object. At the same time a vacuum is applied to outgas and prevent the formation of air bubbles in the blank. The fabricated beryllium blank is rectangular shaped $(800 \mathrm{~mm} \times 800 \mathrm{~mm})$ with a thickness of $40 \mathrm{~mm}$. It is then machined down to a $20 \mathrm{~mm}$ thick spherical blank $\varnothing 800 \mathrm{~mm}$ with a radius of curvature approximately equal to the desired value for the mirror.

The blank (see Fig. 7) is then sent to Kompozit where it is machined down to the final shape and thickness of the mirror and repeatedly annealed for stress relief. The resultant beryllium substrate is $\sim 4 \mathrm{~mm}$ thick and spherically shaped with a radius of curvature very close to its final value. The substrate was not machined down to its design value of $3 \mathrm{~mm}$ thickness because of the considerable risk in breaking it during machining. A radiation hard glue with low outgassing ${ }^{10}$ is used to glue the titanium inserts into the holes of the beryllium substrate rim.

The glass coating is performed at Vavilov. Several small glass sheets are placed on the beryllium substrate front face covering it completely, which is then placed into an oven and heated up to a temperature of $\sim 600{ }^{\circ} \mathrm{C}$ to melt the glass. A single glass layer forms and fuses onto the beryllium substrate. It is then left to cool down slowly. The glass layer is then polished using standard optical methods.

\footnotetext{
${ }^{7}$ Kompozit Joint Stock Company, Moscow (http://www.korolev.ru/english/e_kompozit.html).

${ }^{8}$ Vavilov State Optical Institute, St. Petersburg (http://soi.srv.pu.ru).

${ }^{9}$ Ulba Metallurgical Plant, Ust-Kamenogorsk, Kazakhstan (www.ulba.kz).

${ }^{10}$ Propriety information, special glue used also for space applications.
} 


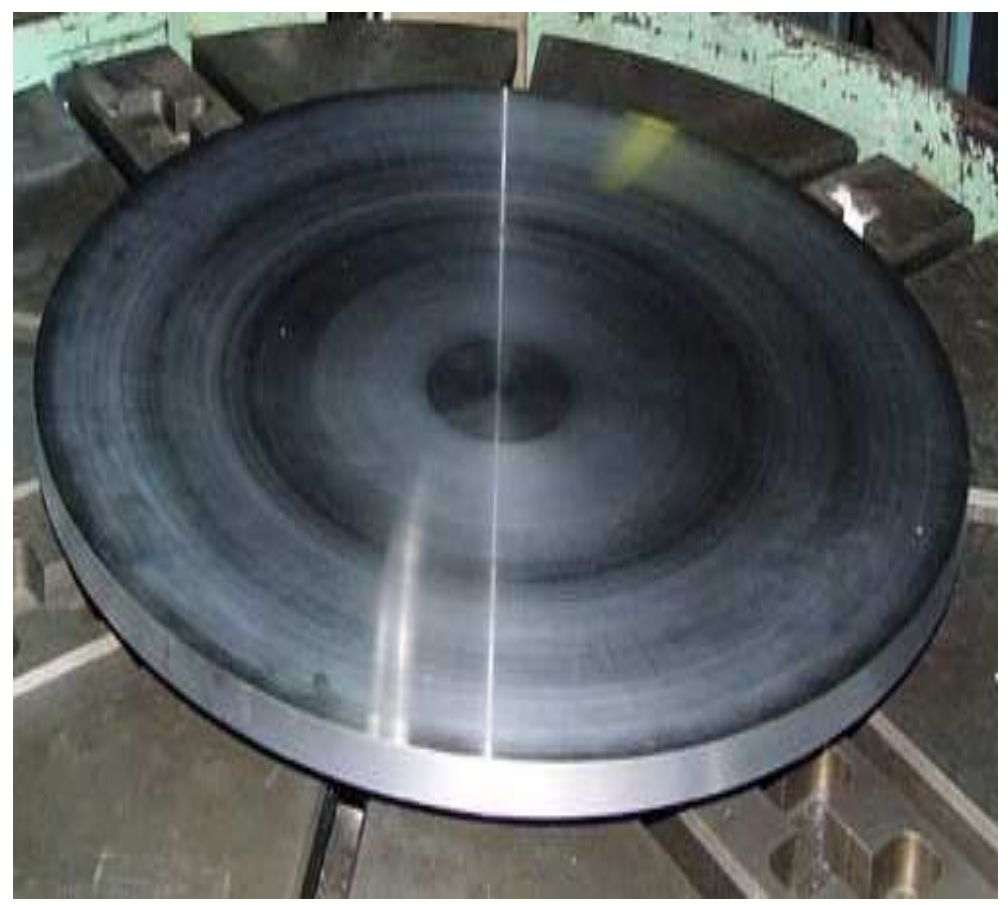

Figure 7: The beryllium blank (20 mm thick, $\varnothing 800 \mathrm{~mm}$ ) at Kompozit before machining.

The glass ${ }^{11}$ and beryllium have nearly the same coefficient of thermal expansion. The average glass thickness after polishing is $0.3-0.5 \mathrm{~mm}$. The fine tuning of the mirror radius of curvature is achieved by the glass polishing, i.e. by varying the thickness of the glass layer across the mirror.

The mirror is returned to Kompozit for final qualification, certification and cleaning to remove any residual beryllium dust, before being delivered to CERN for testing. The uncoated beryllium surface (rim and back side) is passivated by the natural formation of a beryllium oxide surface film due to its exposure to air.

The last step would be the application of a reflective coating ${ }^{12}$ at CERN after the successful testing and consequent acceptance of the mirror. The reflective coating [15] increases the reflectivity to $\gtrsim 85 \%$ for wavelengths $250-500 \mathrm{~nm}$ and $\gtrsim 70 \%$ for wavelengths 200-250 nm. The coating consists of a thin chromium adherence layer followed by an aluminum layer protected by a $\mathrm{SiO}_{2}-\mathrm{HfO}_{2}$ reflective enhancement layer. This type of coating has already been successfully applied onto the surface of the LHCb-RICH2 glass mirrors.

\footnotetext{
${ }^{11}$ The glass composition is propriety information. Its approximate composition is $\mathrm{SiO}_{2} \sim 60 \%$, $\mathrm{CdO} \sim 20 \%, \mathrm{Nb}_{2} \mathrm{O} \sim 15 \%, \mathrm{PbO} \sim 5 \%, \mathrm{~B}_{2} \mathrm{O}_{3} \sim 2 \%, \mathrm{BaO} \sim 1 \%$.

${ }^{12}$ The reflective coating has not yet been applied.
} 


\section{Characterization of the Full-Sized Prototype Mirror}

The mirror was visually inspected and characterized on its arrival at CERN. On visual inspection, several holes and air bubbles were seen in the glass. These are due to a defect in the glass coating process. There are approximately 60 bubbles varying $\sim 0.5-1 \mathrm{~mm}$ in size, concentrated mainly in one sector of the mirror. In addition, the mirror has a large chamfer, up to $\sim 5 \mathrm{~mm}$ from the mirror edge, with thicker glass along the bottom edges (opposite to the rim side). Nevertheless, the resulting optical dead area is very small with the bubbles and chamfer contributing $\sim 0.1 \%$ and $\sim 0.5 \%$ respectively. The mirror radius of curvature is corrected (shortened) by the glass layer, which necessitates a thicker glass layer at the edges. The defects in the glass can be eliminated in future mirrors by refining the glass layering technique and also the glass thickness can be reduced by manufacturing the beryllium substrate with a radius of curvature closer to the final value. The present glass layer could be removed and re-applied to remove the defects.

The mirror was characterized optically, held by a three-point support as shown in Fig. 10. The measured values were $D_{\circ}=3.33 \mathrm{~mm}$ and $\mathrm{R}=2675 \mathrm{~mm}$, shown in plots of Fig. 8 . The mirror was also mounted with the rim at the bottom and vertically tilted by $\sim 13^{\circ}$ (approximate tilt in RICH1), shown in Fig. 9. Within errors the same values for $D_{\circ}$ and $\mathrm{R}$ were measured, proving that the stress and deformations induced in the mirror due to gravity are minimal.
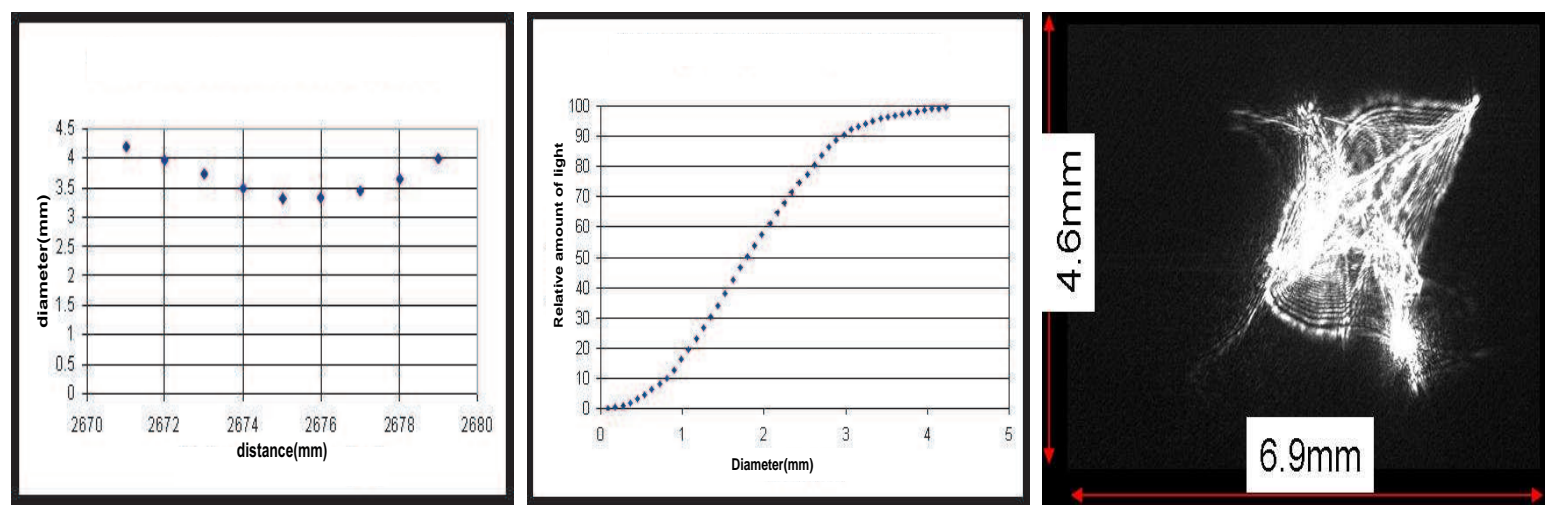

Figure 8: Left plot, the spot size versus the distance of the mirror from the CCD camera; the minimum is for $R=2675 \mathrm{~mm}$ with $D_{\circ}=3.33 \mathrm{~mm}$. Centre plot, the relative amount of light (\%) versus circle diameter for the smallest spot; $95 \%$ is contained by a $\varnothing 3.33 \mathrm{~mm}$ circle. Right photo, the smallest spot i.e. at the mirror centre of curvature. 
The CERN metrology service measured the overall dimensions and thickness of the mirror and it was found to be generally within the specifications. The beryllium substrate with the glass is $\sim 4-5 \mathrm{~mm}$ thick. On average the mirror consists of a $\sim 3.8 \mathrm{~mm}$ thick beryllium substrate with a $\sim 0.4 \mathrm{~mm}$ thick glass layer. The glass layer is thinnest at the centre $(\sim 0.3 \mathrm{~mm})$ gradually increasing in thickness towards the edges up to $\sim 1 \mathrm{~mm}$. A large number of points $(\sim 400)$ were measured on the mirror surface and then fitted to a sphere to extract the mirror radius of curvature and tilt. The metrology extracted value of $\mathrm{R}=2677 \mathrm{~m}$ is close to the optical measurement. Also the extracted angular mirror tilts are within the tolerances of the design specifications given in Fig. 5 and Fig. 6.
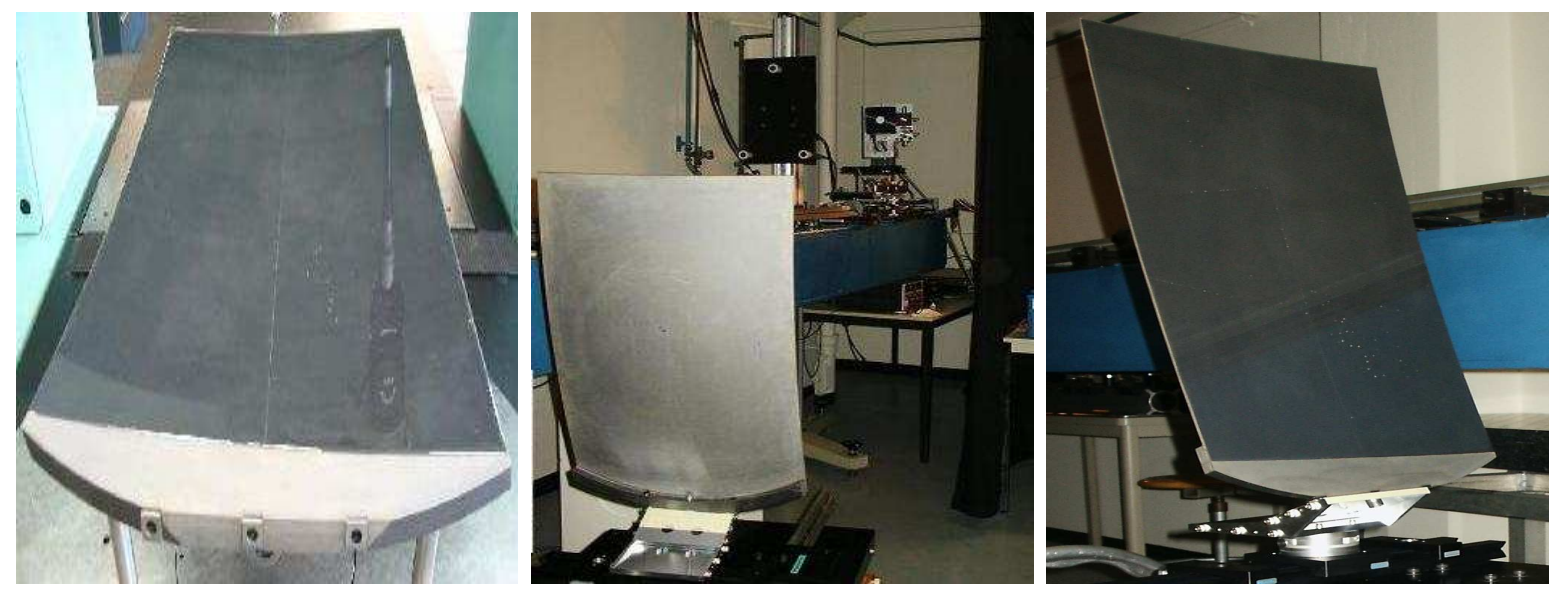

Figure 9: Three photos of the full-sized prototype mirror. Left, a view from above showing the glass coating (dark area) and the rim with the three holes. Centre, a back view of the mirror showing the grey beryllium substrate and the measurement setup. Right, a front view of the mirror showing the glass coating (dark area). In the centre and right photos, the mirror is supported by its rim from the bottom with a single bolt and tilted vertically by $\sim 13^{\circ}$ for the $D_{\circ}$ measurement under the effect of gravity.

A summary of the mirror parameters is given in Table 4. The surface roughness was not measured at CERN but certified by Kompozit to be $<5 \mathrm{~nm}$. The mirror is within the RICH1 specifications, except for the $D_{\text {。 }}$ which should be $<2.5 \mathrm{~mm}$. The thickness of the beryllium substrate was relaxed to $\sim 4 \mathrm{~mm}$ because of the high risk of breaking the beryllium blank during machining. 


\begin{tabular}{|c|c|c|c|c|c|c|c|}
\hline $\begin{array}{c}\text { Dimensions } \\
(\mathrm{mm})\end{array}$ & $\begin{array}{c}\text { Average Thickness } \\
(\mathrm{mm})\end{array}$ & $\begin{array}{c}\mathrm{R} \\
(\mathrm{mm})\end{array}$ & $\begin{array}{c}D_{\circ} \\
(\mathrm{mm})\end{array}$ & $\begin{array}{c}X_{\circ} \\
(\%)\end{array}$ & $\begin{array}{c}\lambda_{I} \\
(\%)\end{array}$ & $\begin{array}{c}\text { Weight } \\
(\mathrm{kg})\end{array}$ & $\begin{array}{c}\text { Roughness } \\
(\mathrm{nm})\end{array}$ \\
\hline $383 \times 660$ & $3.8(\mathrm{Be})+0.4($ glass $)$ & 2675 & 3.33 & $\sim 1.6$ & $\sim 1$ & 2.7 & $<5$ \\
\hline
\end{tabular}

Table 4: Parameters of the full-sized prototype: dimensions (as defined in Fig. 6), average thickness (excluding the rim), the measured $R$ and $D_{\circ}$ values, the average $X_{\circ}$ and $\lambda_{I}$, the total mirror weight, and the surface roughness of the glass. The values for $X_{\circ}$ and $\lambda_{I}$ are estimated. The exact composition of the glass is not known (propriety information).

Unfortunately the project was terminated in January 2006 because of serious uncertainties in the production timescale. A new carbon-fiber composite mirror technology has now been chosen for the RICH1 spherical mirrors.

\section{Conclusions}

Three small sized beryllium-glass prototype mirrors were manufactured and found to be of good optical quality. As a result, a full-sized spherical beryllium mirror was designed and manufactured according to the LHCb-RICH1 specifications. It is the first beryllium-glass mirror ever fabricated with large geometrical dimensions $(\sim 400 \mathrm{~mm} \times 660 \mathrm{~mm})$ but at the same time with a very thin beryllium substrate $(\sim 3.8 \mathrm{~mm})$. Overall the optical quality of the full-sized prototype is good, proving that the technological challenges have been overcome. The full-sized prototype satisfies the RICH1 requirements and can be used as a final RICH1 mirror. A general improvement in the parameters and quality would be expected for future beryllium-glass mirrors by refining the manufacturing technique. 


\section{Appendix}

\section{Mirror Characterization}

The mirror characterization was performed in an optical laboratory at CERN, in a darkroom environment with air circulation and dust filters. The setup for the measurement of spherical converging mirrors is shown in Fig. 10.

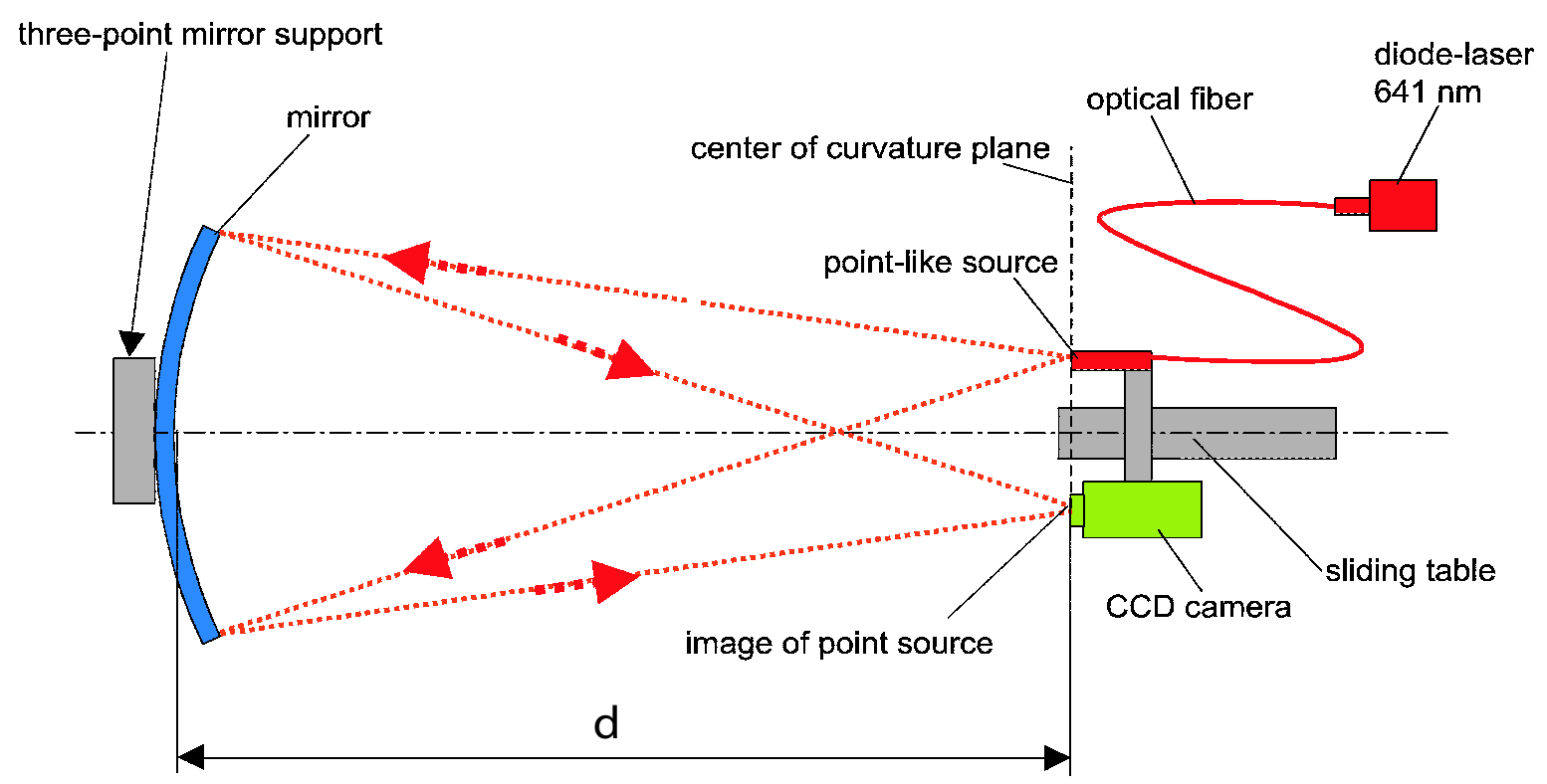

Figure 10: Schematic setup for the radius of curvature $R$ and the $D$ 。 measurements of a spherical converging mirror. The apparatus is mounted on a $10 \mathrm{~m}$ long optical bench.

The mirror to be characterized is held by a three-point support which is mounted on rails. A point-like source is obtained from a diode laser $(\lambda=641 \mathrm{~nm})$ connected to an optical fiber. The laser and a CCD camera ${ }^{13}$ are fixed to a sliding table and move together. The CCD camera is 16 bit, with a pixel size of $9 \mu \mathrm{m} \times 9 \mu \mathrm{m}$ and $6.9 \mathrm{~mm} \times 4.6 \mathrm{~mm}$ sensor size. The sliding table is positioned at a distance $d$ from the mirror, corresponding to the approximate expected radius of curvature of the mirror. The point-like source illuminates uniformly the whole mirror surface and the reflected image (spot) is measured by the CCD camera. The spherical mirror reflects the image of an object, positioned close to its centre of curvature, into an image of the same size and distance from the mirror axis but located on the opposite side of the axis.

${ }^{13}$ DTA model HR400E with a KODAK sensor KAF-400E CCD. 
The measurement is automated. A LabVIEW ${ }^{14}$ program controls both the CCD photograph triggering and the movement of the sliding table using a stepping motor which has a range of $40 \mathrm{~mm}$. The sliding table is moved in steps of $1 \mathrm{~mm}$ across its range, varying the distance $d$ between the mirror and the laser light source. At each $1 \mathrm{~mm}$ step, the CCD takes a photograph of the spot image. At the end of the scan, the LabVIEW program analyzes all the photographs and finds the smallest spot image using the centre of gravity method, from which the mirror $D_{\circ}$ and $R$ are obtained. The radius of curvature $R$ of the mirror is defined as the distance between the mirror reflective surface centre and the CCD sensor for the smallest spot size.

\section{Beryllium Metallurgy}

Beryllium [10] is extracted from its ores through a series of chemical processes and is reduced to pebble form. The pebbles are then vacuum melted and cast into ingots to remove the residual slag. Beryllium metal in cast form is difficult to machine and has poor mechanical properties, therefore powder metallurgy is used to manufacture beryllium objects. Beryllium powder is obtained by chipping the ingots and then mechanically grinding the chips. The ductility and strength of beryllium is related to the grain size; typical grain sizes are $\sim 10-30 \mu \mathrm{m}$ in diameter.

\section{Safety Considerations}

Beryllium is the most toxic of all industrial metals [16]. Beryllium metal in solid form presents no particular health risk. The inhalation of beryllium dust or fumes affects the lungs and poses a serious health risk. Special care is required when handling the beryllium mirror to avoid accidents. Gloves and a mask are recommended by the beryllium safety rules.

\footnotetext{
${ }^{14}$ LabVIEW (Laboratory Virtual Instrumentation Engineering Workbench) is a platform and development environment for a visual programming language from National Instruments.
} 


\section{Acknowledgements}

We thank W. Witzeling for the support and advice in running the beryllium mirrors project with the Russian partners and companies. We also acknowledge A. Cherif and D. Pugnat of the CERN metrology team.

\section{References}

[1] LHCb Collaboration, LHCb RICH TDR, CERN/LHCC/2000-0037, 2000.

[2] LHCb Collaboration, LHCb reoptimized detector design and performance, CERN/LHCC/2003-030, 2003.

[3] S. Easo, Nucl. Instr. and Meth. A 553 (2005) 333, Overview of LHCb RICH detector development.

[4] M. M. Miroshnikov, et al., Optical Engineering 31(04) (1992) 701, Mirrors for optical telescopes.

[5] S. V. Ljubarsky, et al., Proc. SPIE 2199 (1994) 938, Lightweighted mirrors for space telescopes.

[6] Eugene Vygovsky, et al., Proc. SPIE 2543 (1995) 127, Application of Russian beryllium materials in optics.

[7] C. D'Ambrosio, et al., Instr. and Meth. A 478 (2002) 344, Precision optical systems for the new generation of Ring Imaging Cherenkov detectors in high energy physics experiments.

[8] T. Bellunato, et al., Nucl. Instr. and Meth. A 538 (2005) 458, Light composite mirrors for RICH detectors: production, characterisation and stability tests.

[9] Designing with beryllium, Brush Wellman Inc., Cleveland, OH.,USA; www.brushwellman.com.

[10] ASM Handbook, The American Society for Metals, http://www.asm-intl.org.

[11] Review of Particle Physics, Phys. Lett. B 592 (2005) 98.

[12] M. Laub, Development of opto-mechanical tools and procedures for the new generation of RICH-detectors at CERN, Ph.D. Thesis, LHCb 2001-130; Geneva, CERN. 
[13] C. D'Ambrosio, et al., LHCb Public Note, LHCb-2000-071, Geneva, CERN, 12 Apr 2001; The optical systems of LHCb RICHes: a study on the mirror walls and mirrors specifications.

[14] LHCb RICH group, LHCb Public Note, LHCb-2004-121, CERN-LHCb-2004-121, Geneva, CERN, 20 Oct 2005, LHCb RICH1 Engineering Design Review Report.

[15] A. Braem, et al., Nucl. Instr. and Meth. A 553 (2005) 182, Metal multi-dielectric mirror coatings for Cherenkov detectors.

[16] CERN Public Note, Beryllium Safety Instructions IS N ${ }^{0} 25$, EDMS 335747, https://edms.cern.ch/file/335747/1/IS25_E.pdf. 